\title{
Needs and Challenges of Post-Acute Brain Injury Patients in Understanding Personal Recovery
}

\author{
Yamini Karanam, Andrew Miller, and Erin Brady \\ School of Informatics and Computing, Indiana University, Indianapolis \\ 535 W. Michigan St, Indianapolis IN, 46201 \\ \{ykaranam,andrewm,brady\}@iupui.edu
}

\begin{abstract}
Recovery from brain injury is a complex and longitudinal process consisting of clinical recovery, illness management, and personal recovery. Lack of initiatives to create awareness about expected recovery and tools for helping patients understand their progress make the personal recovery journey challenging and frustrating. In this paper, we discuss results from interviews with five post-acute brain injury patients about their needs and challenges in understanding their personal recovery.
\end{abstract}

\section{Keywords}

Brain injury; personal recovery; patient awareness; rehabilitation.

\section{INTRODUCTION}

Brain injury is a structural damage or physiological disruption of brain function and is a leading cause of death and disability around the world. This condition could be a result of external force, illness, stroke, substance abuse, or tumors acquired after birth. It may cause temporary or permanent impairment in areas such as cognitive, emotional, metabolic, motor, perceptual and sensory brain function. In addition, brain injury patients are more susceptible to long-term emotional problems, such as anxiety and depression.

Recovery from brain injury is a complex construct that depends on severity of brain injury, time since injury, and other psychological and social factors. Recovery may not mean that the "suffering has disappeared, all the symptoms removed, and the functioning completely restored" [1]. It is not a linear process, involving more than relief from acute clinical symptoms and occuring even if symptoms reoccur. It changes the frequency and duration of symptoms and is characterized by growth, setbacks and periods of little and rapid change [2]. Side effects from medication, accidents, or other complications arising from brain injury can worsen the frequency and duration of symptoms, hamper recovery, and make understanding the course of recovery unintuitive for patients.

Recovery is also complicated by the diverse communities of practice involved. Different stakeholders and practitioners understand recovery differently, causing a disparity in the understanding of personal recovery among brain injury patients, caregivers, and clinicians which can severely impact rehabilitation $[6,10]$. The medical system currently focuses on and supports clinical recovery (remission as measured by neuroimaging tests and neuropsychological assessments) and symptom-based illness management (including symptom control and long-term symptom monitoring. Less attention is paid to personal recovery, or the holistic process of working toward feeling 'normal again' [2]. A lack of standardized practices for rehabilitation and education

\footnotetext{
Permission to make digital or hard copies of part or all of this work for personal or classroom use is granted without fee provided that copies are not made or distributed for profit or commercial advantage and that copies bear this notice and the full citation on the first page. Copyrights for third-party components of this work must be honored. For all other uses, contact the Owner/Author.
}

ASSETS '17, October 29-November 1, 2017, Baltimore, MD, USA (C) 2017 Copyright is held by the owner/author(s)

ACM ISBN 978-1-4503-4926-0/17/10.

https://doi.org/10.1145/3132525.3134794 leaves patients without awareness of the condition, understanding of expected recovery, and options for rehabilitation [4].

Patients are often unaware of the expected course of personal recovery and its impact on functional domains [8]. Patient selfawareness through an understanding of personal recovery predicts quality of life and impacts rehabilitation outcomes more than the severity of brain injury over time [9]. Thus, there is an urgent need to improve understanding of personal recovery and operationalize recovery for various stakeholders through patient understanding. Patient understanding of personal recovery includes intellectual awareness (knowledge of a particular problem), emergent awareness (recognizing a problem when it occurs), and anticipatory awareness (ability to predict that a problem is likely to occur) [5]. Intellectual awareness provides the foundation for emergent and anticipatory awareness. Factors that lead to impaired intellectual awareness are a lack of adequate information for analyzing past knowledge, impaired abstract reasoning, denial, and self-deception $[5,6]$. The goal of this study is to understand patients' needs and challenges in acquiring adequate information to develop intellectual awareness and enable emergent and anticipatory awareness.

\section{METHODS}

We conducted semi-structured interviews with five post-acute brain injury patients, recruited from an online support group for postsurgery pineal tumor patients. We asked about self-awareness of impairments, similar to the protocol proposed by Fleming et al., and about course of recovery, rehabilitation, tracking and managing symptoms, and participation in clinical decision-making [7].

\section{PRELIMINARY FINDINGS}

All interviews were audio-recorded. We transcribed and analyzed interviews using six-phase essentialist/realist thematic analysis [3]. The age of the participants ranged from $22-54$ years and the duration after surgery ranged from 2 months to 3 years. However, they experienced symptoms few years before diagnosis or surgery.

RQ1: How do patients recovering from brain injury currently track and understand their personal recovery? Lack of clinical or healthcare practices to measure personal recovery leaves the burden of tracking and understanding personal recovery entirely to patients. Our goal is to understand their current practices - data collection instruments, analysis, and insights obtained.

Most participants reported recording pain levels or sleep patterns manually (excel sheet, iPhone app for migraine, paper and pencil) or using a wearable device (Fitbit). The participants explained that logging this information helped them with externalizing the list of symptoms they experience each day and confirm the correlation between symptoms and triggers. P5 spoke about the impact of journaling on reflection and self-awareness. They expressed the need for tangible sources of data that could be used to see trends and communicate their experiences with their healthcare providers.

P5: "I would write in my journal and that has helped me too because when I would go back I would be able to see oh look at 
this, my health was really getting bad here back in August and ... here's the comparison to June you know it was very helpful" "

Participants understood their recovery by self-experimentation and trial and error. They gained an intuitive understanding of their abilities and impairments through observation of daily living.

P2: "I guess just figure then like it was my new normal and avoid anything I could, find my triggers, see any doctor that I could."

RQ2: What are their needs and challenges in this process? Analyzing and reflecting on large amounts of diverse data is challenging without the help of external tools. Our goal is to understand the metaphysical process through which patients gain insights. Through this, we aim to understand what needs and challenges they face in acting on their insights.

Participants stated that the online support group was their main source of information and emotional support. They acquired information about complications of brain injury anecdotally through the stories of other people on the group. They were introduced to the support group either while browsing for information or through another brain tumor survivor. Participants reiterated that the consequences of brain injury and the course of recovery are unique for everyone. Although some of their symptoms were similar to other patients on the support group, the experience of the symptoms and the effect of impairment depends on functional domains such as work, interpersonal relationships etc.

Participants tracked symptoms manually only to discontinue later due to the effort it required.

P1: "Current challenge [is to] chart exactly when it starts. Because there are some days when I wake up with a migraine and obviously I don't know what time it exactly started or what could have caused it".

They believed that data alone did not convey their experience of symptoms without interpretation of how they affected well-being and quality of life.

\section{P3: "The MRI shows that everything is fine I would say I have recovered except for the fatigue issues and double vision."}

RQ3: How is this information used in collaborative care among various stakeholders? We aim to investigate the challenges patients face in the process of communicating an insight to his/her clinician as an input to clinical decision making.

Healthcare providers did not set expectations for setbacks or assess overall well-being. On inquiring, participants were informed by their healthcare providers that they were not informed of the continued complications in order not to cause anxiety about recovery. Patients reported that their healthcare providers' perspective of recovery was very different from their actual recovery. Although they did not conclusively state reasons for this, they speculated that this could be a limitation in the knowledge in the field of neurology. P4 reported that she consulted a neurologist who is a doctor of osteopathy. She reported that his evaluation of her health included well-being while her other doctors who hold M.D.s did not account for it in their assessments. They reported reduced quality of life due to this disparity.

\section{P3: “...unless you had your brain messed with I don't think you} know how it feels... I don't think doctors really get it."

Healthcare providers accounted for recovery from only acute clinical symptoms and set expectations accordingly. Often, they could not anticipate or explain the cause of other complications arising from brain injury. They expected patients to rehabilitate within a few weeks to months. However, participants reported recurring symptoms and new complications preventing them from returning to their pre-injury lives. This simplified perspective of recovery from clinicians may inhibit communication with patients.

Healthcare providers may also be reluctant to use patient generated health data (PGHD) in clinical decision making. P1 was dissuaded from collecting his sleep data first using an excel sheet later with a Fitbit because his healthcare provider refused to use it in validating his concerns. Participants reported that their healthcare providers did not consider this data credible.

P1: "When I logged my sleep patterns in excel for 6-7 months, my doctors refused to even look at it."

\section{CONCLUSIONS AND FUTURE WORK}

Our results show that most participants tracked their symptoms using personal memoirs, paper or mobile based trackers, or wearable devices. However, they understand their overall recovery intuitively. Healthcare providers are reluctant to use the insights obtained through this process due to issues with validity and reliability. Given its individualistic and complicated nature, we believe that personal health informatics has the potential for leveraging the use of patient generated health data in patients' everyday lives and clinician encounters. In future work, we will conduct longitudinal studies collecting data about health-related quality of life to capture a patient's perception of symptoms and functional status. This data is useful to quantify the degree to which a disease or treatment impacts a patient's life in a valid and reliable way. We will use it to co-design and evaluate exploratory visualizations iteratively to enable reflection and self-awareness in patients and evaluate how visualization tools facilitate selfadvocacy and participation in clinical decision making for patients.

\section{REFERENCES}

[1] Anthony, W.A. 1993. Recovery from mental illness: The guiding vision of the mental health service system in the 1990s. Psychosocial Rehabilitation Journal. 16, 4 (1993), 1123.

[2] Barber, M.E. 2012. Recovery as the new medical model for psychiatry. Psychiatric Services (Washington, D.C.). 63, 3 (Mar. 2012), 277-279.

[3] Braun, V. and Clarke, V. 2006. Using thematic analysis in psychology. Qualitative Research in Psychology. 3, 2 (Jan. 2006), 77-101.

[4] Cioe, N. 2012. Factors influencing post-acute brain injury rehabilitation treatment outcome. Dissertations. (May 2012).

[5] Crosson, B. et al. 1989. Awareness and compensation in postacute head injury rehabilitation. Journal of Head Trauma Rehabilitation. 4, 3 (Sep. 1989), 46-54.

[6] Flashman, L.A. and McAllister, T.W. 2002. Lack of awareness and its impact in traumatic brain injury. Neurorehabilitation. 17, 4 (2002), 285-296.

[7] Fleming, J.M. et al. 1996. Self-awareness of deficits in adults with traumatic brain injury: how best to measure? Brain Injury. 10, 1 (Jan. 1996), 1-16.

[8] Pagulayan, K.F. et al. 2006. A Longitudinal Study of HealthRelated Quality of Life After Traumatic Brain Injury. Archives of Physical Medicine and Rehabilitation. 87, 5 (May 2006), 611-618.

[9] Soberg, H.L. et al. 2013. Health-related Quality of Life 12 months after severe traumatic brain injury: A prospective nationwide cohort study. Journal of Rehabilitation Medicine. 45, 8 (Sep. 2013), 785-791.

[10] Stotland, N.L. et al. 2008. The recovery concept: clinician and consumer perspectives. Journal of Psychiatric Practice. 14 Suppl 2, (May 2008), 45-54. 